\title{
Usefulness of Serum Omentin-1 Levels for the Prediction of Adverse Cardiac Events in Patients with Hypertrophic Cardiomyopathy
}

\author{
Suleyman Sezai Yıldız ${ }^{a} \quad$ Irfan Sahin $^{b} \quad$ Gokhan Cetinkal $^{\mathrm{a}}$ Gokhan Aksan $^{\mathrm{a}}$ \\ Suat Hayri Kucuk ${ }^{c}$ Kudret Keskin ${ }^{a}$ Sukru Cetin ${ }^{a}$ Serhat Sigirci ${ }^{a}$ \\ Illhan Illker Avcl ${ }^{d} \quad$ Hakan Kilci $^{a} \quad$ Kadriye Kiliçkesmez ${ }^{a}$
}

${ }^{a}$ Department of Cardiology, Sisli Hamidiye Etfal Training and Research Hospital, Istanbul, Turkey; ${ }^{b}$ Department of Cardiology, Bagcilar Training and Research Hospital, Istanbul, Turkey; ${ }^{\mathrm{C}}$ Department of Clinical Biochemistry, Bagcilar Training and Research Hospital, Istanbul, Turkey; ${ }^{\mathrm{d}}$ Department of Cardiology, Siyami Ersek Cardiovascular and Thoracic Surgery Center, Istanbul, Turkey

\section{Significance of the Study}

- This study investigated the association between serum omentin-1 levels and adverse cardiac events in patients with hypertrophic cardiomyopathy (HCM). Serum omentin-1 levels were lower in patients with HCM than in healthy controls and were associated with adverse cardiac events during the 2-year follow-up. Therefore, the serum omentin-1 level could serve as a biochemical marker to predict the prognosis of patients with HCM.

\section{Keywords}

Omentin · Hypertrophic cardiomyopathy · Adverse cardiac events

\begin{abstract}
Objective: To investigate the association between serum omentin-1 levels and adverse cardiac events in patients with hypertrophic cardiomyopathy (HCM). Subjects and Methods: This prospective, observational study included 87 patients with HCM and 50 age- and sex-matched control subjects. Serum omentin-1 and brain natriuretic peptide (BNP) levels were measured in all subjects, using enzyme-linked immunosorbent assay and electrochemiluminescence, respectively. Patients with HCM were divided into 2 groups ac-
\end{abstract}

cording to their omentin levels, i.e., low: $\leq 291 \mathrm{ng} / \mathrm{mL}(n=48)$ and high: $>291 \mathrm{ng} / \mathrm{mL}(n=39)$. Cardiac mortality, hospitalization due to heart failure, and implantable cardioverterdefibrillator (ICD) implantation were considered adverse cardiac events. Statistical analysis included uni- and multivariant logistic regression, receiver-operating characteristic (ROC) analysis, and the Kaplan-Meier method. Results: Serum omentin-1 levels were significantly lower in the obstructive $(253.9 \pm 41.3 \mathrm{ng} / \mathrm{mL})$ and nonobstructive $(301.9 \pm 39.8$ $\mathrm{ng} / \mathrm{mL}$ ) HCM groups than in the control group (767.1 \pm 56.4 $\mathrm{ng} / \mathrm{mL}), p<0.001$, respectively. The BNP levels were higher in the obstructive and nonobstructive HCM groups than in the control group $(269.5 \pm 220,241.0 \pm 227$, and $24.0 \pm 18.9$ $\mathrm{pg} / \mathrm{mL}$, respectively, $p<0.001)$. The Kaplan-Meier analysis indicated that patients with low omentin-1 levels showed a

\section{KARGER}

E-Mail karger@karger.com www.karger.com/mpp
(C) 2018 The Author(s) Published by S. Karger AG, Basel

Karger Open access

This is an Open Access article licensed under the Creative Commons Attribution-NonCommercial-4.0 International License (CC BY-NC) (http://www.karger.com/Services/OpenAccessLicense), applicable to the online version of the article only. Usage and distribution for commercial purposes requires written permission.
Suleyman Sezai Yildız

Department of Cardiology, Sisli Hamidiye Etfal Training and Research Hospital Halaskargazi Caddesi, Etfal Sokak

TR-34371 Sisli-İstanbul (Turkey)

E-Mail sezai04@yahoo.com 
significantly higher (48.2\%) 2-year cumulative incidence of overall adverse cardiac events than those with high omentin-1 levels (16.2\%) (log-rank test, $p=0.001)$. In the multivariate logistic regression analysis, omentin-1, interventricular septum (IVS) thickness, and male gender were independent predictors of adverse cardiac events in the follow-up. Conclusion: Omentin-1 levels were lower in patients with HCM than in the control group, and this was associated with worse cardiac outcomes.

(c) 2018 The Author(s)

Published by S. Karger AG, Basel

\section{Introduction}

Hypertrophic cardiomyopathy (HCM) is the most common genetic cardiovascular disease, affecting approximately 1 in every 500 adults $(0.2 \%)$ [1]. It is inherited in an autosomal dominant manner, and is characterized by a wall thickness $\geq 15 \mathrm{~mm}$ in $\geq 1$ left ventricular (LV) myocardial segments (that cannot be explained by abnormal overloading conditions) [2]. HCM is a myocardial disease that presents with LV hypertrophy, myocardial fibrosis, and myocardial irregularity [3]. In previous studies, it has been shown that chronic inflammation decreases myocardial contractility and induces hypertrophy, apoptosis, and fibrosis $[4,5]$. The natural course of HCM is highly variable, ranging from being asymptomatic throughout life, to progressive heart failure (HF), and premature sudden cardiac death (SCD) early in life [6].

Schaffler [7] identified omentin as an anti-inflammatory adipokine that is predominantly expressed and secreted by visceral adipose tissue rather than by subcutaneous adipose tissue. Omentin plays a pivotal role in adipose tissue differentiation, maturation, metabolism, immune response regulation, inflammation, and insulin resistance $[8,9]$. Negative and positive correlations between serum levels of omentin-1 and various conditions have been reported [10-12]. Huang et al. [13] reported a negative correlation between nonischemic dilated cardiomyopathy and circulating levels of omentin-1. Narumi et al. [14] reported that decreased serum levels of omentin-1 were associated with poor cardiac outcome in patients with HF.

However, whether or not serum omentin-1 levels are associated with clinical outcomes in patients with HCM remains unclear. Therefore, the objective of this study was to investigate the relationship between serum levels of omentin-1 and cardiac prognosis in patients with HCM.

\section{Subjects and Methods}

\section{Patients and Study Protocol}

This prospective, observational study included 87 patients with HCM and 50 age- and sex-matched controls without HCM admitted to the Cardiology Outpatient Clinic, Bagcilar Training and Research Hospital, Istanbul, Turkey, in the period March 2012 to January 2015. The patients with HCM were followed for 2 years.

HCM was diagnosed based on the definition of the European Society of Cardiology, i.e., a wall thickness of $\geq 15 \mathrm{~mm}$ in $\geq 1 \mathrm{LV}$ myocardial segments, as measured by any imaging technique that is not explained solely by loading conditions [2]. Exclusion criteria were coronary artery disease $(n=4)$, a symptoms-consistent New York Heart Association (NYHA) class of $>3(n=4)$, cardiac valvular disease $(n=4)$, uncontrolled hypertension (a blood pressure of $>140 / 90 \mathrm{~mm} \mathrm{Hg}$ with or without medications; $n=7)$, active or chronic inflammatory disease $(n=3)$, aortic valve stenosis $(n=$ 2 ), and severe renal failure (estimated glomerular filtration rate of $\left.<30 \mathrm{~mL} / \mathrm{min} / \mathrm{m}^{2} ; n=1\right)$. Thirty-seven subjects were enrolled in the obstructive HCM group, 50 in the nonobstructive HCM group, and 50 in the control group. The medical history, risk factors, a family history of SCD, medication use, and NYHA class of all the subjects involved in the study were recorded. Body mass index (BMI) was calculated (by G.C., G.A., and S.S.). Each patient was assessed based on physical examinations and electrocardiography done every 3 months, and 24-h ambulatory electrocardiography was performed at least once (by S.S.Y., İ.S., S.S., S.C., and İ.I.A.). Patients who reported symptoms of chest pain, dyspnea, palpitation, dizziness, and syncope were reevaluated using echocardiography and 24-h ambulatory electrocardiography; based on the outcome, treatment was planned in an outpatient or inpatient setting. An implantable cardioverter-defibrillator (ICD) was implanted (by S.S.Y., G.A., and K.K.) in patients who had survived cardiac arrest due to ventricular fibrillation or sustained ventricular tachycardia that caused syncope or hemodynamic compromise. In the follow-up, 2 cardiologists (H.K. and G.C.), who were blinded to the data of blood biomarkers, reviewed the medical records of the patients and made phone calls to patients or their relatives to find out if cardiovascular events had occurred. Rate of hospitalization, syncope, status according to NYHA class, mortality, and arrhythmic events were recorded. Hospitalizations due to HF, ICD implantation, and cardiac death were considered as adverse cardiac events.

The study was performed in accordance with the recommendations of the Declaration of Helsinki on Biomedical Research Involving Human Subjects. The Bagcilar Training and Research Hospital Ethics Committee approved the study protocol and written informed consent was obtained from each patient.

\section{Echocardiography Study}

Two-dimensional (2-D), M-mode, and Doppler echocardiographic studies based on the recommendations of the American Society of Echocardiography/European Association of Echocardiography [15] were performed using an ultrasonography machine (IE33; Philips Medical Systems, Andover, MA, USA). The maximum wall thickness measured at any border of the LV was considered the maximal thickness. The diameter of the left atrium (LA), diastolic interventricular septum (IVS), and LV posterior wall thickness were recorded from the parasternal short- and longaxis views. The peak velocity of the early $(\mathrm{E})$ and late $(\mathrm{A})$ waves and 
Table 1. The clinical, demographic, and biochemical characteristics of the study population

\begin{tabular}{|c|c|c|c|c|}
\hline & $\begin{array}{l}\text { Obstructive HCM } \\
(n=37)\end{array}$ & $\begin{array}{l}\text { Nonobstructive HCM } \\
(n=50)\end{array}$ & $\begin{array}{l}\text { Control } \\
(n=50)\end{array}$ & $p$ value \\
\hline Age, years & $40.9 \pm 12.1$ & $36.6 \pm 13$ & $37.7 \pm 9.6$ & 0.35 \\
\hline Male/female, $n$ & $20 / 17$ & $25 / 25$ & $24 / 26$ & 0.81 \\
\hline Hypertension & $5(13.5)$ & $3(10.3)$ & $3(8.3)$ & 0.3 \\
\hline Hypercholesterolemia & $4(10.8)$ & $5(10.0)$ & $4(8.0)$ & 0.3 \\
\hline Family history of HCM & $7(18.9)^{*}$ & $9(18.0)^{*}$ & $0(0)$ & 0.005 \\
\hline Family history of SCD & $2(5.4)^{*}$ & $2(4.0)^{*}$ & $0(0)$ & 0.1 \\
\hline Previous AF & $5(13.5)$ & $6(12.0)$ & $0(0)$ & 0.13 \\
\hline BMI & $25.7 \pm 3.4$ & $25.2 \pm 3.2$ & $24.0 \pm 3.4$ & 0.1 \\
\hline Fasting blood glucose, $\mathrm{mg} / \mathrm{dL}$ & $98.3 \pm 24.6$ & $95.9 \pm 15.7$ & $89.9 \pm 13.6$ & 0.32 \\
\hline $\mathrm{HbA}_{1 \mathrm{c}}, \%$ & $5.6 \pm 0.7$ & $5.5 \pm 0.4$ & $5.3 \pm 0.3$ & 0.26 \\
\hline Creatinine, $\mathrm{mg} / \mathrm{dL}$ & $0.85 \pm 0.2$ & $0.82 \pm 0.2$ & $0.81 \pm 0.1$ & 0.08 \\
\hline $\mathrm{TG}, \mathrm{mg} / \mathrm{dL}$ & $135.0 \pm 18.0$ & $140.0 \pm 18.0$ & $136.0 \pm 17.0$ & 0.09 \\
\hline LDL-C, mg/dL & $130.0 \pm 21.0$ & $136.0 \pm 25.0$ & $129.0 \pm 21.0$ & 0.4 \\
\hline HDL-C, mg/dL & $45.0 \pm 8.0$ & $43.0 \pm 8.0$ & $47.0 \pm 9.0$ & 0.08 \\
\hline BNP level, pg/mL & $269.5 \pm 224.4^{*}$ & $241.0 \pm 217.6^{*}$ & $24.0 \pm 18.9$ & $<0.001$ \\
\hline Omentin-1 level, ng/mL & $253.9 \pm 41.3^{*, \#}$ & $301.9 \pm 39.8^{*}$ & $767.1 \pm 56.4$ & $<0.001$ \\
\hline \multicolumn{5}{|l|}{ Medication } \\
\hline Amiodarone & $9(24.3)^{*, \#}$ & $5(10.0)^{*}$ & $0(0)$ & 0.005 \\
\hline Warfarin & $4(10.8)$ & $4(8.0)$ & $0(0)$ & 0.13 \\
\hline$\beta$-Blocker & $31(83.8)^{*, \#}$ & $29(58.0)^{*}$ & $0(0)$ & $<0.001$ \\
\hline ACE-I/ARB & $4(14.3)$ & $2(6.9)$ & $2(5.6)$ & 0.09 \\
\hline
\end{tabular}

Data are expressed as mean \pm SD or $n(\%)$, unless otherwise indicated. ACE-I, angiotensin-converting enzyme inhibitor; $\mathrm{ARB}$, angiotensin receptor blocker; $\mathrm{AF}$, atrial fibrillation; $\mathrm{BNP}$, brain natriuretic peptide; HCM, hypertrophic cardiomyopathy; $\mathrm{HbA}_{1 \mathrm{c}}$, glycated hemoglobin; HDL-C, high-density lipoprotein cholesterol; LDL-C, low-density lipoprotein cholesterol; SCD, sudden cardiac death; TG, triglycerides. ${ }^{*} p<0.01$ versus control group, ${ }^{\#} p<0.05$ versus group 2 .

the E/A ratio were calculated from the transmitral flow velocity, using an apical 4-chamber view and positioning the sample volume at the tip of the mitral leaflets during diastole. The septal early diastolic velocity (e') of the mitral annulus from the apical 4 -chamber view was measured using tissue Doppler imaging. The $\mathrm{LV}$ diastolic function was defined as the ratio of $\mathrm{E}$ to e velocity (E/e'). The LV volume and ejection fraction were computed using the biapical Simpson rule. The dynamic LV outflow tract (LVOT) pressure gradient was measured using continuous-wave Doppler in the apical views, either while resting or while being provoked like in the Valsalva maneuver. LVOT obstruction was defined as the presence of a peak instantaneous LVOT gradient of $\geq 30 \mathrm{~mm}$ $\mathrm{Hg}$. All echocardiographic examinations were recorded and analyzed at the end of the study by 2 independent, experienced echocardiographers (İ.İ.A. and S.S.), who were blinded to the subjects' clinical characteristics and levels of serum omentin-1 and brain natriuretic peptide (BNP).

\section{Laboratory Measurements}

Peripheral venous blood samples were collected from the antecubital veins of all the subjects after $12 \mathrm{~h}$ of fasting, and centrifuged at 3,000 rpm for $10 \mathrm{~min}$. The serum was then separated and stored at $-80^{\circ} \mathrm{C}$ until analysis. Levels of serum fasting blood glucose, creatinine, blood urea nitrogen, triglycerides (TG), total cholesterol
(TC), low-density lipoprotein cholesterol (LDL-C), and high-density lipoprotein cholesterol (HDL-C) were measured using standard laboratory techniques (Hitachi 7600 Automatic Biochemical Analyzer, Hitachi Co., Japan). Serum levels of omentin-1 were determined using a commercially available enzyme-linked immunosorbent assay (ELISA) kit with high sensitivity and specificity for detecting human omentin-1 (Sunred Biotecnology Co., Shangai, China). Serum BNP levels were assessed using the electrochemiluminescence ECLIA method (Roche Cobas 8000 Modular Analyzer System, Roche Diagnostics, UK).

\section{Statistical Analysis}

Statistical analysis was performed using SPSS v21 software (SPSS Inc., Chicago, IL, USA). Data are reported as mean \pm SD for continuous variables. Categorical variables are reported as percentages. The normality assumption was evaluated using the Kolmogorov-Smirnov test. Continuous variables in the 3 groups were compared using a one-way analysis of variance or the KruskalWallis test. Continuous variables were compared between 2 groups using the independent-samples $t$ test or the Mann-Whitney U test. Categorical data were compared using the $\chi^{2}$ or Fisher exact test. Univariate logistic regression analysis was performed, and the variables with a $p$ value $<0.1$ were then entered into a backward stepwise multivariate logistic regression model to assess the 


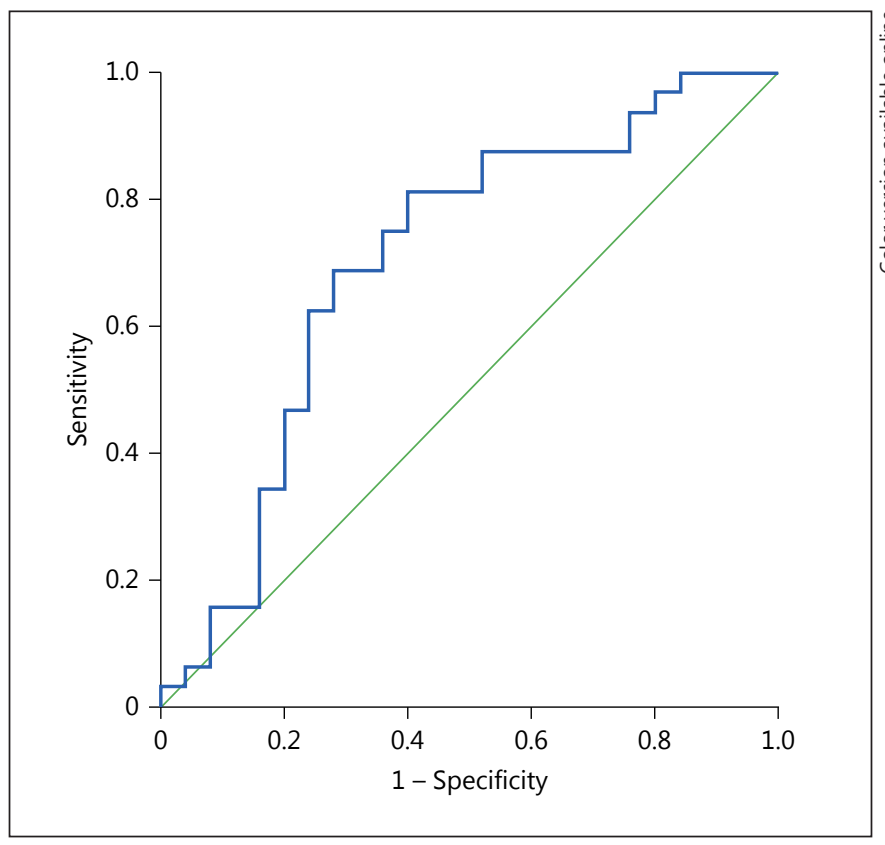

Fig. 1. In the ROC curve analysis, an omentin-1 level $\leq 291 \mathrm{ng} / \mathrm{mL}$ had a sensitivity of $69 \%$ and a specificity of $75 \%$ in predicting adverse events in patients with hypertrophic cardiomyopathy (AUC $0.780,95 \%$ CI $0.56-0.85, p=0.009$ ).

independent predictors of adverse cardiac events in HCM. Eventfree survival curves were generated using the Kaplan-Meier method. Differences in survival curves among the low and high omentin-1 subgroups were assessed using the log-rank test. Receiveroperating characteristics (ROC) analysis was used to determine the sensitivity, specificity, and positive and negative predictive values of serum levels of omentin-1 for adverse cardiac events during the long-term follow-up. Based on the ROC analysis, we divided the patients into 2 groups according to cut-off values as follows: a low omentin ( $\leq 291 \mathrm{ng} / \mathrm{mL})$ and high omentin (>291 ng/ $\mathrm{mL}$ ). A post hoc power analysis was performed with a sample size of 137, using the mean values and SDs of the omentin levels of the subjects in the patient and control groups and an $\alpha$ level set at $p<$ 0.05 . The effect size of the study population was 0.80 , and the power of the study was $0.93 . p<0.05$ was considered statistically significant.

\section{Results}

The clinical, demographic, and laboratory findings of the patients are given in Table 1 . No significant differences were found among the groups regarding BMI, hypertension, and hypercholesterolemia. Levels of creatinine, glycated hemoglobin, LDL-C, HDL-C, TG, and fasting glucose were similar in both groups. Serum levels of omentin-1 levels were significantly lower in the obstruc-

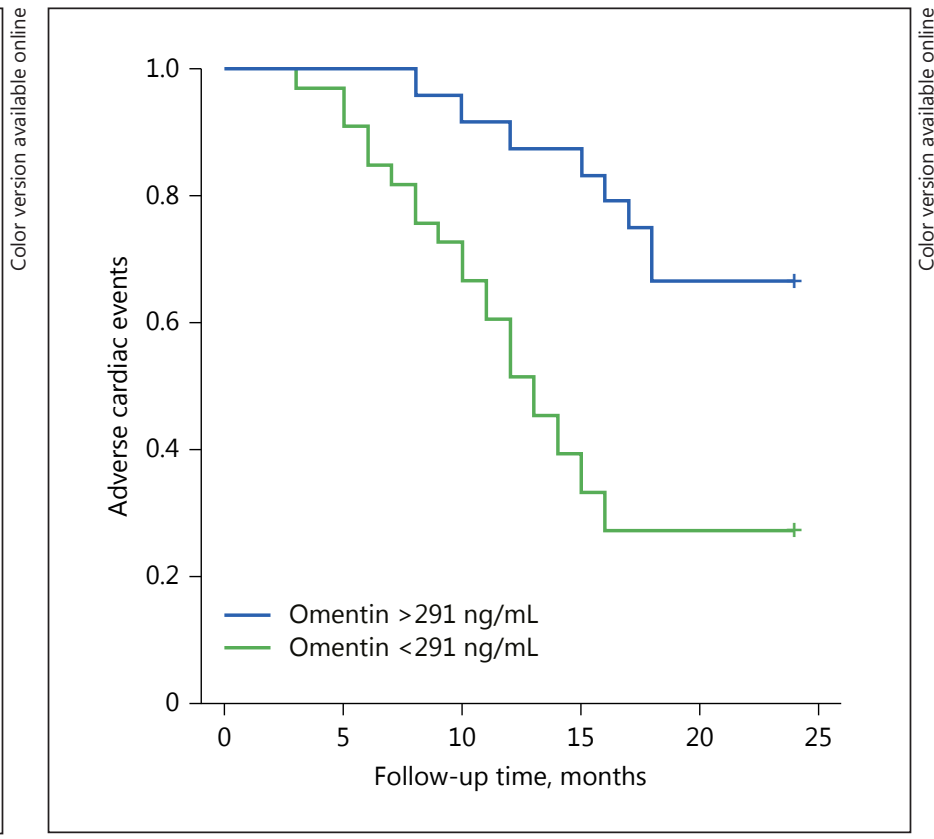

Fig. 2. In the Kaplan-Meier curve analysis, the rate of cardiac adverse events was significantly higher in the low omentin-1 group than in the high omentin-1 group (log-rank test, $p<0.001$ ).

tive and nonobstructive HCM groups than in the control group $(253.9 \pm 41.3,301.9 \pm 39.8,767.1 \pm 56.4 \mathrm{ng} / \mathrm{mL}$, respectively, $p<0.001)$. Furthermore, the obstructive HCM group had the lowest serum levels omentin- 1 between groups $(253.9 \pm 41.3$ vs. $301.9 \pm 39.8$ vs. $767.1 \pm 56.4$ $\mathrm{ng} / \mathrm{mL}, p<0.001)$. The level of BNP was significantly higher in the obstructive and nonobstructive HCM groups than in the control group $(269.5 \pm 224.4,241.0 \pm 217.6$, and $24.0 \pm 18.9 \mathrm{pg} / \mathrm{mL}$, respectively, $p<0.001$ ). However, no significant difference in the serum level of BNP was found between obstructive and nonobstructive HCM.

Using ROC curve analysis, omentin levels of $\leq 291 \mathrm{ng} /$ $\mathrm{mL}$ were predictive of adverse cardiac events in HCM patients, with $75 \%$ specificity and $69 \%$ sensitivity (area under the curve [AUC] $0.780,95 \%$ confidence interval [CI] $0.56-0.85, p=0.009$; Fig. 1). Characteristics of patients with low and high omentin levels are shown in Table 2. At baseline, no difference in LV systolic and diastolic diameters, posterior wall thickness, LVOT peak gradient, and LV ejection fraction were found between the low and high omentin groups (Table 2). The patients with low serum levels of omentin- 1 had a thicker IVS $(2.8 \pm 0.5$ vs. $2.0 \pm 0.6 \mathrm{~cm}, p<0.001)$ and larger LA ( $3.9 \pm 0.4$ vs. $3.3 \pm$ $0.5 \mathrm{~cm}, p<0.001)$ than the high omentin group. In addition, the E/e' ratio was significantly higher $(p<0.001)$ and 
Table 2. The clinical, demographic, and echocardiographic characteristics of the study population according to low and high serum omentin-1 levels

\begin{tabular}{|c|c|c|c|}
\hline & $\begin{array}{l}\text { Low omentin } \\
\leq 291 \mathrm{ng} / \mathrm{mL}(n=48)\end{array}$ & $\begin{array}{l}\text { High omentin } \\
\geq 291 \mathrm{ng} / \mathrm{mL}(n=39)\end{array}$ & $p$ value \\
\hline Age, years & $40.3 \pm 12.4$ & $36.5 \pm 10.5$ & 0.12 \\
\hline Male/female, $n$ & $28 / 20$ & $22 / 17$ & 0.92 \\
\hline Body mass index & $26.4 \pm 4.1$ & $25.8 \pm 3.4$ & 0.16 \\
\hline Family history of SCD & $3(6.2)$ & $1(2.5)$ & 0.01 \\
\hline Atrial fibrillation & $6(12.5)$ & $5(12.8)$ & 0.68 \\
\hline NYHA class >1 & $14(42.4)$ & $4(12.5)$ & 0.004 \\
\hline Syncope & $10(20.5)$ & $7(18.0)$ & 0.07 \\
\hline Heart failure & $14(29.2)$ & $6(15.4)$ & 0.02 \\
\hline Nonsustained VT & $11(22.9)$ & $9(23.1)$ & 0.9 \\
\hline Sustained VT & $4(8.3)$ & $3(7.7)$ & 0.6 \\
\hline Hospitalization & $18(37.5)$ & $5(12.8)$ & $<0.01$ \\
\hline ICD implantation & $9(18.7)$ & $3(7.7)$ & 0.02 \\
\hline Mortality & $3(6.2)$ & $1(2.5)$ & 0.04 \\
\hline Adverse cardiac events & $23(47.9)$ & 7 (17.9) & $<0.001$ \\
\hline Ejection fraction, \% & $62.3 \pm 4.2$ & $61.3 \pm 3.8$ & 0.38 \\
\hline IVS thickness, cm & $2.8 \pm 0.5$ & $2.0 \pm 0.6$ & $<0.001$ \\
\hline PW thickness, cm & $1.9 \pm 0.3$ & $1.9 \pm 0.4$ & 0.9 \\
\hline LA diameter, $\mathrm{cm}$ & $3.9 \pm 0.4$ & $3.3 \pm 0.5$ & $<0.001$ \\
\hline LV diastolic diameter, $\mathrm{cm}$ & $4.5 \pm 0.4$ & $4.6 \pm 0.4$ & 0.43 \\
\hline LV systolic diameter, $\mathrm{cm}$ & $3.07 \pm 0.4$ & $3.13 \pm 0.4$ & 0.63 \\
\hline LVOT peak gradient, $\mathrm{mm} \mathrm{Hg}$ & $36 \pm 18$ & $32 \pm 20$ & 0.5 \\
\hline E wave, $\mathrm{cm} / \mathrm{s}$ & $68.9 \pm 16.6$ & $75.7 \pm 6.5$ & $<0.001$ \\
\hline A wave, $\mathrm{cm} / \mathrm{s}$ & $77.4 \pm 16.3$ & $63 \pm 14$ & $<0.001$ \\
\hline Septal e' wave, $\mathrm{cm} / \mathrm{s}$ & $6.0 \pm 2.4$ & $9.2 \pm 2.5$ & $<0.001$ \\
\hline E/e'ratio & $13.2 \pm 3.9$ & $9.0 \pm 3.0$ & $<0.001$ \\
\hline
\end{tabular}

Data are expressed as mean \pm SD or $n(\%)$, unless otherwise indicated. A wave, peak late transmitral filling velocity; E' wave, peak early diastolic mitral annulus velocity on tissue Doppler imaging; E wave, peak early transmitral filling velocity; ICD, implantable cardioverter defibrillator; LA, left atrium; LV, left ventricle; LVOT, left ventricular outflow tract; IVS, interventricular septum; NYHA, New York Heart Association; PW, posterior wall; SCD, sudden cardiac death; VT, ventricular tachycardia.

the septal e'-wave $(p<0.001)$ and E/A ratio $(p<0.001)$ significantly lower in the low omentin group than in the high omentin group.

During 24 months of follow-up, sustained and nonsustained ventricular tachycardia (detected using Holter monitoring) as well as syncope were found to be similar in the low omentin and high omentin groups $(4[8.3 \%], 3$ [7.7\%], $p=0.6 ; 11$ [22.9\%], 9 [23.1\%], $p=0.9 ; 10$ [20.8\%], 7 [18.0\%], $p=0.07$, respectively; Table 2). The number of patients with NYHA class $>1$ and the rate of HF were higher in the low omentin group than in the high omentin group (14 [29.2\%], 4 [10.2\%], $p<0.01$, and 14 [29.2\%], 6 [15.4\%], $p=0.02$, respectively). Hospitalizations due to HF, ICD implantation, and cardiac mortality rates were higher in the low omentin group than in the high omentin group (18 [37.5\%], 5 [12.8\%], $p<0.01 ; 9$ [18.7\%], 3 [7.7\%], $p=0.02$; and 3 [6.2\%], 1 [2.5\%], $p=0.04$; respectively). The composite end point of hospitalization due to HF, ICD implantation, and cardiac mortality occurred in 23 $(47.9 \%)$ patients in the low omentin group and 7 (17.9\%) patients in the high omentin group $(p<0.001)$. KaplanMeier curves revealed that the low omentin group had a significantly higher prevalence (48.2\%) of adverse cardiac events than the high omentin group (16.2\%) (log-rank test, $p<0.001$; Fig. 2).

The univariate logistic regression analysis revealed that serum levels of BNP, the LVOT peak gradient, the IVS thickness, male sex, a family history of SCD, and serum levels of omentin- 1 showed a trend $(p<0.1)$ towards an association with adverse cardiac events in HCM. The multivariate logistic regression analysis demonstrated that serum levels of omentin-1, IVS thickness, and male 
Table 3. Univariate and multivariate analysis of HCM patients for predicting adverse cardiac events

\begin{tabular}{|c|c|c|c|c|}
\hline & \multicolumn{2}{|l|}{ Univariate } & \multicolumn{2}{|l|}{ Multivariate } \\
\hline & HR (95\% CI) & $p$ value & HR (95\% CI) & $p$ value \\
\hline Omentin-1 level & $14.3(4.96-35.1)$ & $<0.001$ & $5.10(3.5-9.9)$ & $<0.01$ \\
\hline BNP level & $5.1(1.81-8.06)$ & 0.03 & & \\
\hline Creatinine & $1.78(0.54-5.83)$ & 0.34 & & \\
\hline Hypertension & $0.69(0.32-1.83)$ & 0.43 & & \\
\hline LVOT gradient & $4.6(2.03-9.1)$ & 0.03 & & \\
\hline IVS thickness & $11.1(3.9-30.9)$ & $<0.001$ & $3.85(2.2-8.1)$ & 0.02 \\
\hline NYHA class $>1$ & $0.94(0.20-4.32)$ & 0.93 & & \\
\hline Male gender & $7.36(1.2-15.7)$ & 0.01 & $2.57(1.8-7.7)$ & 0.02 \\
\hline A family history of SCD & $4.04(1.38-11.8)$ & 0.01 & & \\
\hline Age & $0.99(0.96-1.04)$ & 0.93 & & \\
\hline
\end{tabular}

$\mathrm{HR}$, hazard ratio; $\mathrm{CI}$, confidence interval; BNP, brain natriuretic peptide; HCM, hypertrophic cardiomyopathy; IVS, interventricular septum; LVOT, left ventricular outflow tract; NYHA, New York Heart Association; SCD, sudden cardiac death.

sex were independent predictors of a poor prognosis for HCM (hazard ratio [HR] 5.10, 95\% CI 3.5-9.9, $p<0.01$; HR 3.85, 95\% CI 2.2-8.1, $p=0.02$; and HR 2.57, 95\% CI $1.8-6.7, p=0.02$; respectively; Table 3 ).

\section{Discussion}

In this study, serum levels of omentin- 1 were lower in patients with HCM than in controls. HCM patients with lower serum omentin-1 levels $(\leq 291 \mathrm{ng} / \mathrm{mL})$ had a larger LA, higher LVOT gradient, thicker IVS, and greater diastolic dysfunction than HCM patients with higher serum omentin-1 levels (>291 ng/mL). A serum level of omentin- $1 \leq 291 \mathrm{ng} / \mathrm{mL}$ was a predictor of a poor prognosis, and involved cardiac mortality, hospitalization due to HF, and ICD implantation during the 24-month followup, with a sensitivity of $69 \%$ and specificity of $75 \%$. A negative correlation was observed between serum levels of omentin-1 and BNP in patients with HCM. To our knowledge, there is no study in the literature investigating the association between serum levels of omentin-1 and adverse cardiac events in patients with HCM.

Hypertrophy of the myocardium, diminished coronary blood flow, microvascular dysfunction, and chronic inflammation all act together in the pathophysiology of HCM, ultimately leading to myocardial fibrosis which is the central pillar of the disease [16-18]. In previous studies, C-reactive protein, TNF- $\alpha$, and IL- 6 , which are all inflammatory biomarkers in HCM, were found to be related to the pathogenesis of the disease $[17,19]$. In addition, BNP, as a biomarker of wall tension, and myocardial fibrosis have been shown to increase in patients with HCM compared to normal subjects [20].

We did not study inflammatory biomarkers in our study, as our main objectives were to analyze the serum levels of omentin-1 and BNP and investigate whether these levels had an effect on cardiac events. We found that, compared to controls, BNP levels were elevated 11fold and 10-fold in patients with obstructive and nonobstructive HCM, respectively.

Adipose tissue performs many endocrine functions via its production and secretion of various bioactive peptides, called adipokines [21]. Recent studies have shown that some adipokines play an active role in preventing cardiovascular diseases owing to their anti-inflammatory, antioxidant, and antiapoptotic properties [22, 23]. Omentin-1 belongs to this group of adipokines, and is mainly produced in the stromal vascular cells of visceral adipose tissue [24]. It is negatively correlated with chronic inflammation, endothelial dysfunction, arterial stiffness, atherosclerosis, and calcification $[25,26]$. Recently, Narumi et al. [14] showed that low omentin-1 (but not BNP) was an independent predictor of cardiac events in patients with HF due to different etiologies in a 1-year follow-up. Likewise, Wang et al. [27] reported that serum levels of omentin-1 were reduced in patients with ischemic HF, and they found a negative correlation between the serum level of omentin-1 and the severity of HF. 
In our study, we also found that decreased omentin-1 was associated with poor prognosis for patients with HCM. Patients with a higher NYHA class had lower serum concentrations of omentin-1 than those with a lower NYHA class, a finding which could indicate that reduced omentin-1 might be associated with HF severity. Although serum levels of omentin-1 were not affected by LVOT gradient, patients with low omentin-1 had greater IVS thickness and worse diastolic dysfunction than the high omentin group.

We speculate that low serum omentin levels correlate negatively with cardiac filling pressures, LV diastolic dysfunction, and abnormal LV wall stress. We also suggest that patients with low serum omentin-1 levels have higher myocardial inflammation and fibrosis. Thus, in our study, serum omentin-1 was an independent predictor of adverse cardiac events. However there was no association between serum BNP and cardiac outcomes. Omentin-1 could contribute to the anti-inflammatory process that leads to the slowing down of myocardial fibrosis and the prevention of future adverse cardiac events in patients with HCM. Therefore, serum omentin levels may be considered for the diagnosis of unfavorable LV remodeling which predicts adverse cardiac events.
The limitations of this study were its relatively small sample size and it being conducted at a single center. In addition, cardiac MRI was not used to detect the presence and the extent of myocardial fibrosis. Also, concentrations of other adipocytokines and inflammatory markers were not measured. Future studies with larger sample sizes are required to elucidate the relationship between circulating levels of omentin- 1 and the prognosis of patients with HCM.

\section{Conclusion}

In this study, serum levels of omentin were lower in patients with obstructive and nonobstructive HCM, and this was associated with worse cardiac outcomes. Therefore, serum omentin-1 might be a new biomarker for predicting myocardial fibrosis and the development of adverse cardiac events in patients with HCM.

\section{Disclosure Statement}

There were no conflicts of interest.

\section{References}

1 Maron BJ, Gardin JM, Flack JM, et al: Prevalence of hypertrophic cardiomyopathy in a general population of young adults. Echocardiographic analysis of 4,111 subjects in the CARDIA study. Coronary Artery Risk Development in (Young) Adults. Circulation 1995; 92:785-789.

2 Elliott PM, Anastasakis A, Borger MA, et al: 2014 ESC guidelines on diagnosis and management ofhypertrophic cardiomyopathy: the Task Force for the Diagnosis and Management of Hypertrophic Cardiomyopathy of the European Society of Cardiology (ESC). Eur Heart J 2014;35:2733-2779.

3 Richardson P, Mckenna W, Bristow M, et al: Report of the 1995 World Health Organization/ International Society and Federation of Cardiology Task Force on the Definition and Classification of Cardiomyopathy. Circulation 1996;93:841-842.

4 Nian M, Lee P, Khaper N, et al: Inflammatory cytokines and postmyocardial infarction remodeling. Circ Res 2004;94:1543-1553.

5 Aukrust P, Gullestad L, Ueland T, et al: Inflammatory and anti-inflammatory cytokines in chronic heart failure: potential therapeutic implications. Ann Med 2005;37:74-85.
6 Maron BJ, Casey SA, Poliac LC, et al: Clinical course of hypertrophic cardiomyopathy in a regional United States cohort. JAMA 1999; 281:650-655.

7 Schäffler A, Neumeier M, Herfarth $H$, et al: Genomic structure of human omentin, a new adipocytokine expressed in omental adipose tissue. Biochim Biophys Acta 2005;1732:96102.

8 Jaikanth C, Gurumurthy P, Cherian KM, et al: Emergence of omentin as a pleiotropic adipocytokine. Exp Clin Endocrinol Diabetes 2013; 121:377-383.

9 Zhou JY, Chan L, Zhou SW: Omentin: linking metabolic syndrome and cardiovascular disease. Curr Vasc Pharmacol 2014;12:136-143.

10 de Souza Batista CM, Yang RZ, Lee MJ, et al: Omentin plasma levels and gene expression are decreased in obesity. Diabetes 2007;56: 1655-1661.

11 Pan HY, Guo L, Li Q: Changes of serum omentin-1 levels in normal subjects and in patients with impaired glucose regulation and with newly diagnosed and untreated type 2 diabetes. Diabetes Res Clin Pract 2010;88: 29-33.
12 Saremi A, Asghari M, Ghorbani A: Effects of aerobic training on serum omentin- 1 and cardio metabolic risk factors in overweight and obese men. J Sports Sci 2010;28:993-998.

13 Huang Y, Lin Y, Zhang S, et al: Circulating omentin-1 levels are decreased in dilated cardiomyopathy patients with overt heart failure. Dis Markers 2016;2016:6762825.

14 Narumi T, Watanabe T, Kadowaki S, et al: Impact of serum omentin-1 levels on cardiac prognosis in patients with heart failure. Cardiovasc Diabetol 2014,13:84.

15 Lang RM, Badano LP, Mor-avi V, et al: Recommendations for cardiac chamber quantification by echocardiography in adults: an update from the American Society of Echocardiography and the European Association of Cardiovascular Imaging. J Am Soc Echocardiogr 2015;28:1-39.

16 Ahn HS, Kim HK, Park EA, et al: Coronary flow reserve impairment in apical vs asymmetrical septal hypertrophic cardiomyopathy. Clin Cardiol 2013;36:207-216.

17 Kuusisto J, Karja V, Sipola P, et al: Low-grade inflammation and the phenotypic expression of myocardial fibrosis in hypertrophiccardiomyopathy. Heart 2012;98:1007-1013. 
18 O'Hanlon R, Grasso A, Roughton M, et al: Prognostic significance of myocardial fibrosis in hypertrophic cardiomyopathy. J Am Coll Cardiol 2010;56:867-874.

19 Högye M, Mándi Y, Csanády M, et al: Comparison of circulating levels of interleukin-6 and tumor necrosis factor-alpha in hypertrophic cardiomyopathy and in idiopathic dilated cardiomyopathy. Am J Cardiol 2004;94: 249-251.

20 Maron BJ, Tholakanahalli VN, Zenovich AG, et al: Usefulness of B-type natriuretic peptide assay in the assessment of symptomatic state in hypertrophic cardiomyopathy. Circulation 2004;109:984-989.
21 Mojiminiyi OA, Al Mulla F, Abdella NA: Which obesity index best explains the link between adipokines, coronary heart disease risk and metabolic abnormalities in type 2 diabetes mellitus? Med Princ Pract 2009;18:123129.

22 Ouchi N, Parker JL, Lugus JJ, et al: Adipokines in inflammation and metabolic disease. Nat Rev Immunol 2011;11:85-97.

23 Zhang Y, Wang XL, Zhao J, et al: Adiponectin inhibits oxidative/nitrative stress during myocardial ischemia and reperfusion via PKA signaling. Am J Physiol Endocrinol Metab 2013;305:1436-1443.
24 Frankel DS, Vasan RS, D’Agostino RB Sr, et al: Resistin, adiponectin, and risk of heart failure: the Framingham Offspring study. J Am Coll Cardiol 2009;53:754-762.

25 Moreno-Navarrete JM, Ortega F, Castro A, et al: Circulating omentin as a novel biomarker of endothelial dysfunction. Obesity 2011;19: 1552-1559.

26 Yoo HJ, Hwang SY, Hong HC, et al: Association of circulating omentin-1 level with arterial stiffness and carotid plaque in type 2 diabetes. Cardiovasc Diabetol 2011;10:103.

27 Wang XH, Dou LZ, Gu C, et al: Plasma levels of omentin-1 and visfatin in senile patients with coronary heart disease and heart failure. Asian Pac J Trop Med 2014;7:55-62. 